\title{
Enhancement Of Nuclear Reactions Due To Screening Effects Of Core Electrons
}

\author{
N. LUO, P. J. SHRESTHA AND G. H. MILEY \\ Department of Nuclear, Plasma and Radiological Engineering, University of Illinois. \\ 103 S. Goodwin, \\ Urbana, IL 61801, USA \\ E-mail:nluo@uiuc.edu \\ V. VIOLANTE \\ Associazione Euratom-ENEA sulla Fusione, Centro Ricerche Frascati, \\ C.P. 65 - 00044 Frascati, \\ Rome, Italy
}

\begin{abstract}
Recent progress in understanding the screening effects of core level atomic electrons is summarized in this paper. Some preliminary results on core electron screening were reported before [1]. The studies focus on two types of nuclear reactions in some metal lattices: fusion between deuterons and also proton capture by medium and heavy lattice nuclei. In both reactions the energy of the light nuclear species, proton or deuteron, is on the $\mathrm{KeV}$ (1000 electron volts) scale, while that of heavy nuclei is essentially zero. A standard atomic code is used to obtain the core electron charge density and the potential profile in the metal atom. This Hartree-Fock-Slater type code was originally written by Herman and Skillman [2] and later modified by others and available online [3]. For the D-D reaction, the charge density obtained then gives an estimate on the screening length. The corresponding enhancement in Coulomb barrier tunneling can be obtained from this data. For the proton capture reaction, an ion dynamic code [4] written to simulate the motion of $\mathrm{KeV}$ protons in Pd/Ni lattice, CLAIRE, was modified to take into account the realistic atomic potential, including core electron contributions. In both cases, our result shows a significant nuclear reaction enhancement. The reaction rate calculated roughly matches the scale of excess heat observed in some metal hydride/deuteride [5] experiments.
\end{abstract}

\section{Introduction}

The nuclear fusion probability of two hydrogen isotopes is known to be limited by the repulsive Coulomb barrier. This is especially true in the ordinary hot plasma condition where the hydrogen isotopes are fully ionized. Each isotopic $\mathrm{H}^{+}$(including $\mathrm{D}^{+}$and $\mathrm{T}^{+}$) feels the full Coulomb potential of another, which gives rise to the well-known Gamow exponential factor [1], and strongly reduces the nuclear fusion reaction rate.

In a solid state environment, the situation could be quite different. The Coulomb screening of electrons has to be considered in this case. In general, when a positive charge is immersed in a Fermi sea of electrons (with negative charge of course), the electrons respond by accumulating around the positive charge and therefore partially screen out its positive Coulomb potential. The enormous effect of electron screening on nuclear reaction rate has recently been firmly established [6-8]. It is notable to see these efforts come from diverse disciplines, covering fields from nuclear engineering to astrophysics. This diverse nature of the research adds further credibility to their results.

Although the experiments have proved the significance of electron screening, a theoretical explanation is still far from satisfactory: the screening effect is far beyond that expected from the band electrons along. The authors think that the difference may be due to the contribution of core electrons. In a suitable condensed matter system, such as a metal, the electrons may come either from the valence levels or from the core levels, depending upon 
the energy state of $\mathrm{H}$ nucleus: only those with enough energy of over a few hundred electron volts will be able to reach the core level. It is therefore rather intuitive that a higher electron density will result in better screening effect. Therefore, the screening effect of core level electrons, generally neglected in the literature of fusion research, is focus of the present study. In fact, in experiments such as LUNA [6-8], the metal targets are bombarded by D beams of a few thousand electron volts, and there should be no problem for deuterons of such an energy scale to reach the core level. Indeed those experiments have demonstrated a significant enhancement in D-D fusion rate at those low bombarding energies.

In this paper, a realistic atomic potential and charge density of a typical metal, palladium, are obtained by using a numerical atomic-structure calculation method. This charge density is then used to calculate the screening effect, which in turn determines the fusion reaction rate.

\section{Model and Calculation}

To the first order approximation, the Thomas-Fermi screening wave-vector [9] is given by

$$
q_{T F}=\left(\frac{6 \pi N e^{2}}{E_{f}}\right)^{1 / 2},
$$

where $N$ is the volume density of electron and the $E_{f}$ the Fermi energy of the electron system, including valence electrons and core electrons up to $L$-shell, which in the case of a metal is on the order of a few eV.

When a positive charge $e_{c}$ is placed in an electron sea with density $N$, its electrostatic potential felt by a test charge is no longer a pure Coulomb potential but rather a screened one, as given by the following Yukawa form,

$$
V=\frac{e_{c} e^{-q_{T F} r}}{4 \pi \varepsilon r}
$$

The exponential factor in Eq. (2) strongly damps the potential to virtually zero after a few Thomas-Fermi lengths as defined by

$$
l_{T F}=\frac{1}{q_{T F}}
$$

A Hartree-Fock-Slater (HFS) code [3] is used to calculate the realistic atomic potential and density. There are a few different ways to deal with atomic structures, HFS being one of the simplest. HFS basically utilizes a local density approximation (LDA) and is the ancestor of modern density functional theory (DFT) atomic codes. Strictly speaking, the latest DFT codes or the Multi-Configuration Hartree-Fock (MCHF) atomic packages, will give better accuracy, but here our main objective is for illustration and an estimate of the order of magnitude. In this regard the HFS code should be adequate, and it was employed here.

The calculated atomic charge density for Pd is plotted in Fig. 1, from which a Thomas-Fermi screening length is readily calculated and shown in Fig. 2. The atomic potential is given in Fig. 3 


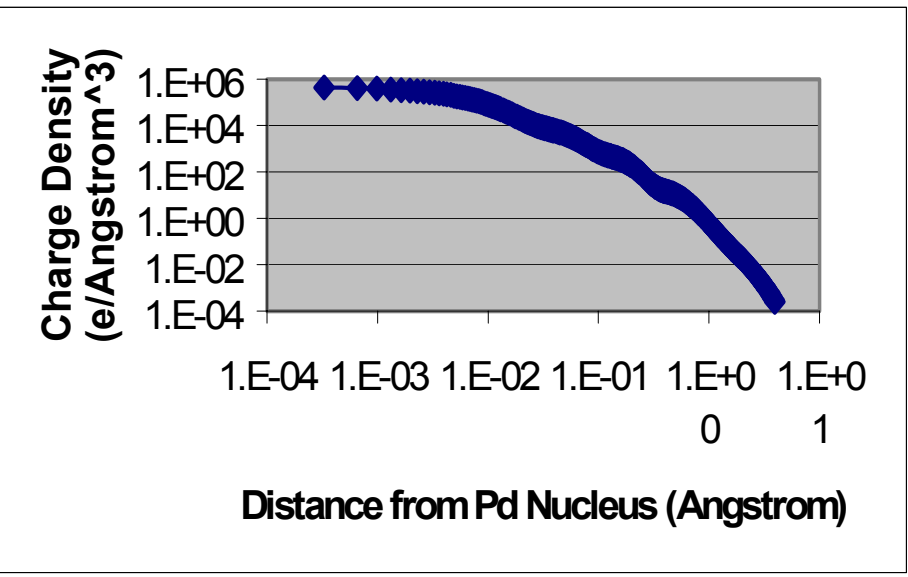

Figure 1. Electron charge density in Pd atom.

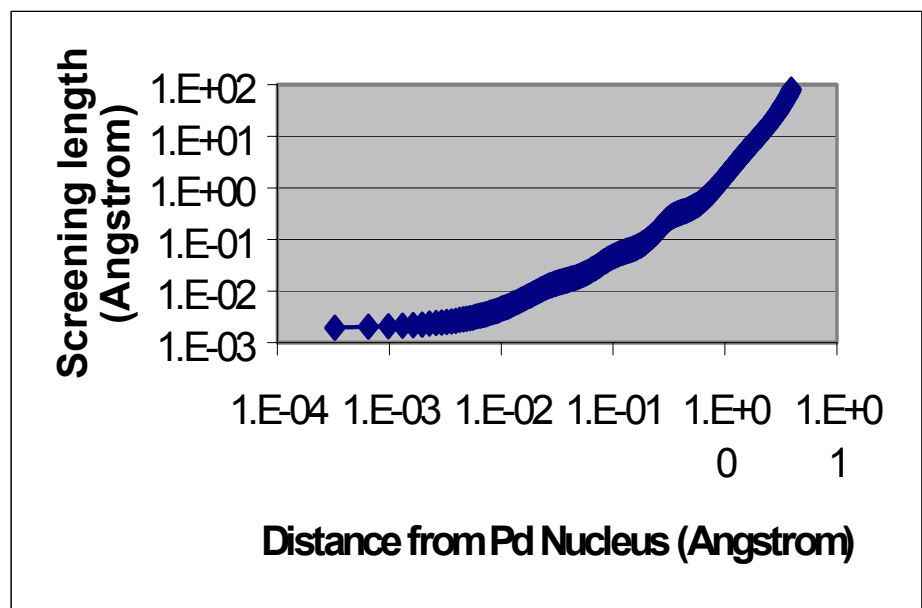

Figure 2. Electron screening length in Pd atom.

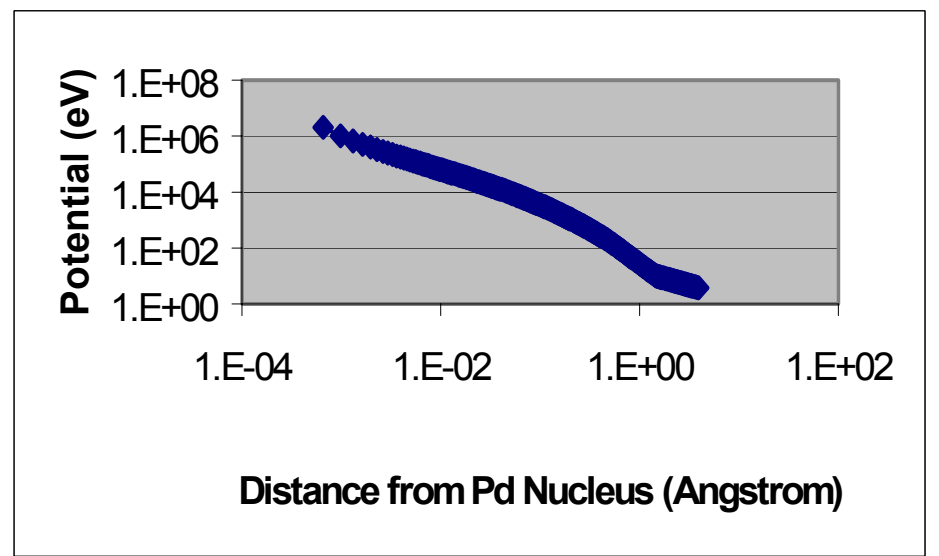

Figure 3. Atomic potential distribution in $\mathrm{Pd}$ atom.

\section{Application to D-D and proton capture reaction}

Now that the atomic potential and screening length are obtained versus the distance from the Pd nucleus, the enhancement of nuclear reaction can be addressed for both the D-D and the proton capture reactions. 


\subsection{D-D Fusion Reaction}

D-D fusion proceeds mainly through the following routes, with a roughly equal branching ratio:

$D+D=T+P$

$D+D=N+{ }^{3} H e$.

As a rule-of-thumb, the D-D nuclear fusion has a reasonable probability of occuring when the distance between two isotopic $\mathrm{H}$ nuclei is reduced to the picometer scale. Judging from Fig. 2, such a condition is readily satisfied by the high electron density of $L$-shell electrons in $\mathrm{Pd}$ : If a deuteron is 2.4 picometer away from a Pd nucleus, the screening length is already reduced to below 1 picometer.

Of course a penalty occurs in energy in order to gain screening: It takes some extra energy for $\mathrm{H}$ nuclei to reach the $\mathrm{Pd} L$-level because of the Coulomb repulsion between $\mathrm{Pd}$ and D. However this does not require large amount of energy as manifested in Fig. 3. Only $3 \mathrm{KeV}$ is required to reach the picometer-scale radius of $L$-shell.

Now we consider the fusion cross-section between two deuterons of $1 \mathrm{KeV}$ each, when both are subject to core electron screening. From Fig. 3 a deuteron can reach 0.1 angstrom within the Pd nucleus. The corresponding screening length at that distance is roughly 0.04 angstrom. Using this screened potential, the tunneling rate can be calculated by the following WKB approximation:

$$
\Gamma=e^{-\int_{r}^{0} \frac{\sqrt{2 m(V-E)}}{\hbar} d r}
$$

Note that this formula is only for one dimension. For three-dimension, it always underestimates the tunneling rate and can serve as a lower bound for the reaction crosssection. From (4) the screened D-D fusion cross-section is bound below by $1 \times 10^{-4}$ Barns. The value for non-screened (plasma phase) $\mathrm{D}-\mathrm{D}$ is often quoted as $1 \times 10^{-9}$ Barns [10]. Therefore there is at least a 5 orders of magnitude increase in the fusion cross-section once the core electrons are taken into account.

\subsection{Proton Capture Reaction}

There have been numerous reports of low energy nuclear reactions between the proton and the host metals such as Pd [11]. Next we want to investigate the possibility of such an interaction.

Of course here a much higher Coulomb barrier, which comes from the Pd nucleus, is present. Fortunately, the increase in the Coulomb barrier is partly offset by the presence of 46 electrons: the realistic atomic potential seen around a $\mathrm{Pd}$ nucleus is much weaker than otherwise with a pure nuclear Coulomb potential.

The CLAIRE code [4] was written to simulate collision events between a proton and a Pd lattice atom. Of course under equilibrium and static situations the collisions would be fairly rare or of very small energy; so nuclear events of any sort would be highly unlikely. CLAIRE therefore assumes that crucial non-equilibrium conditions are established by outside excitations such as electrolysis, and such conditions induce a coherent oscillation, similar to oscillations observed in solid state plasma. The protons in the presence of such a plasma can undergo acceleration and gain an energy up to a few thousand electron volts. 
CLAIRE was initially written with only a pure Coulomb potential in mind. Therefore it is easy to understand that the result would underestimate the penetration power of protons. In fact the previous simulation never shows that the proton can reach under 0.1 angstroms of the Pd nucleus. Now that a realistic atomic Pd potential has been obtained, a modification of CLAIRE was carried out to include this potential in the current study. This atomic potential is fit by an analytical formula and used in CLAIRE in place of the pure Pd nuclear Coulomb one. The modification significantly changes the result. Fig. 4 shows results of a typical simulation. Now protons routinely reach the Pd nuclei at a picometer scale. Fig. 4 is a result of a typical simulation.

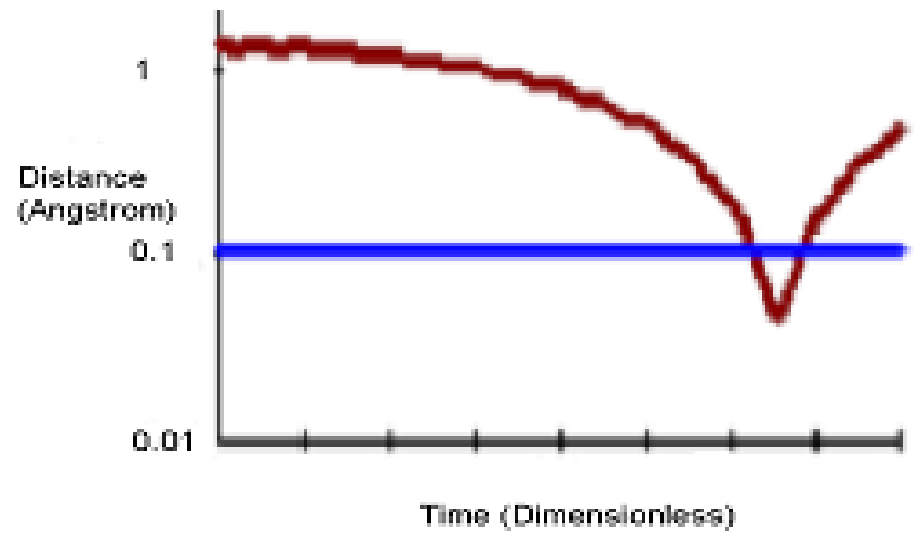

Figure 4. Proton-Pd distance versus time in a typical collision.

As mentioned before, a closest distance on the picometer scale signifies a Coulomb barrier tunneling rate significant to observe nuclear reactions, matching that that needed to explain prior excess heat observation [5]. Therefore the acceleration mechanism embedded in CLAIRE's physics, combined with a realistic consideration of the atomic charge density and potential distribution, offer a possible explanation for the proton-metal nuclear reaction suggested by various LENR experiments.

\section{Discussion}

Our calculations show unambiguously that various nuclear reactions ensue in a solid state environment as long as either deuterons or protons attain a kinetic energy of $\mathrm{KeV}$ level. A question then naturally arises: how could the hydrogen isotopes gain such a large energy (in ordinary solid state scale) in the first place?

The required oscillation could originate from a solid-state plasma effect when $\mathrm{PdH}$ is driven by external non-equilibrium forces, such as for example, an electric current. It is known that electrolytic process is prone to some sorts of electromagnetic instability and oscillation [12]. This actually is not a surprise at all considering the following effects: 1) the metal-liquid interface is well know to be a capacitive double layer. 2) the metal-electrolyte interface could work as a heavy-doped tunneling diode when the electrolyte is highly concentrated, or as a Schottcky diode when the electrolyte is dilute. In either case a nonlinear device is present. 3) $\mathrm{PdD}(\mathrm{H})$ has well-known decreasing resistivity when the $\mathrm{D}(\mathrm{H})$ loading is beyond a certain range. This resembles a negative differential resistivity effect [13]. These three effects combined make possible an oscillator, just as those widely used in various electromagnetic radiation sources. As long as the oscillation happens, an energetic, nonequilibrium solid-state plasma becomes possible. 


\section{Conclusions}

The atomic charge distribution is shown to play an important role in low energy nuclear reactions in solid state environment, as verified by the model calculation results presented here. Further studies using more sophisticated atomic structure methods, such as the MCHF code, are highly desired in the future.

\section{Acknowledgments}

This research was supported by Lattice Energy, LLC.

\section{References}

1. N. Luo, G. H. Miley and A. G. Lipson, Transactions of The American Nuclear Societ, 88 (2003) 622.

2. F. Herman, S. Skillman, Atomic Structure Calculations, Prentice-Hall, Englewood Cliffs, NJ, (1963).

3. http://hermes.phys.uwm.edu/projects/elecstruct/hermsk/codes/HS.CodesAvail.html

4. V. Violante, A. Torre, G. Selvaggi and G. H. Miley, Fusion Technology, 39 (2000) 266.

5. C. H. Castano, et al., ICCF-9 Proceedings (X. Z. Li, editor) (2002) 24.

6. F. Strieder, C. Rolfs, C. Spitaleri, and P.Corvisiero, Naturwissenschaften 88 (2001) 461.

7. H. Yuki, J.Kasagi, A.G.Lipson, T.Ohtsuki, T.Baba, T.Noda, B.F.Lyakhov and N.Asami, JETP Lett. 68 (1998) 11.

8. F. Raiola et al., Physics Letters B 547 (2002) 193.

9. P. Nozieres, D. Pines, The Theory of Quantum Liquids, Perseus Books, Cambridge, MA, p. 150 (1999).

10. G.H. Miley, H. Towner and N. Ivich, Fusion Cross Section and Reactivities, Rept. COO2218-17, University of Illinois, Urbana, IL, 1974.

11. G.H. Miley and P. J. Shrestha, this Proceedings.

12. H. Helms, E. Schlomer and W. Jansen, Monatshefte Fur Chemie, 129 (1998) 617.

13. N. Luo and G.H. Miley, this Proceedings. 